\title{
Coulomb vs. physical string tension on the lattice
}

\author{
Giuseppe Burgio, Markus Quandt, Hugo Reinhardt, and Hannes Vogt \\ Institut für Theoretische Physik, Auf der Morgenstelle 14, 72076 Tübingen, Germany
}

(Dated: September 12, 2018)

\begin{abstract}
From continuum studies it is known that the Coulomb string tension $\sigma_{C}$ gives an upper bound for the physical (Wilson) string tension $\sigma_{W}[1$. How does however such relationship translate to the lattice? In this paper we give evidence that there, while the two string tensions are related at zero temperature, they decouple at finite temperature. More precisely, we show that on the lattice the Coulomb gauge confinement scenario is always tied to the spatial string tension, which is known to survive the deconfinement phase transition and to cause screening effects in the quark-gluon plasma. Our analysis is based on the identification and elimination of center vortices which allows to control the physical string tension and study its effect on the Coulomb gauge observables. We also show how alternative definitions of the Coulomb potential may sense the deconfinement transition; however a true static Coulomb gauge order parameter for the phase transition is still elusive on the lattice.
\end{abstract}

\section{INTRODUCTION}

Recent years have seen a rising interest in Coulomb gauge investigations of Yang-Mills theories in general, and in the Hamiltonian formulation in particular [2, 18]. In the latter, once Weyl-gauge is implemented to eliminate the $A_{0}(x)$ components of the gauge fields, the Hamilton operator and the Gauß's law constraint are invariant under the residual time-independent gauge transformations and, moreover, only depend on the remaining space-like gauge fields and momenta $\boldsymbol{A}^{a}(\boldsymbol{x}), \hat{\boldsymbol{\Pi}}^{a}(\boldsymbol{x})$. In Abelian theories the transversal part of these vector fields is gauge-independent and thus physical, so that Coulomb gauge can be seen as the physical gauge, eliminating all gauge-dependent degrees of freedom. In non-Abelian theories this is no longer strictly true, but the elimination of the longitudinal degrees of freedom via Coulomb gauge still resolves Gauß' law, providing a formulation in terms of the transversal fields ${ }^{1} \boldsymbol{A}_{\perp}^{a}, \boldsymbol{\Pi}_{\perp}^{a}$ alone, where studies of the Yang-Mills ground state are more natural. Such a resolution of Gauß' law through Coulomb gauge thus "automatically" incorporates the constraints one should impose in the Hamiltonian formulation, circumventing the explicit construction of the physical Hilbert space [19]. This results in:

$$
\begin{aligned}
H & =H_{\mathrm{G}}+H_{\mathrm{C}} \\
H_{\mathrm{G}} & =\int d^{3} x\left[\frac{1}{2} \mathcal{J}^{-1}[\boldsymbol{A}] \Pi_{i}^{a} \mathcal{J}[\boldsymbol{A}] \Pi_{i}^{a}+\frac{1}{4} F_{i j}^{a} F_{i j}^{a}\right] \\
H_{\mathrm{C}} & =\frac{g^{2}}{2} \int d^{3}(x, y) \mathcal{J}^{-1}[\boldsymbol{A}] \rho^{a}(\boldsymbol{x}) \mathcal{J}[\boldsymbol{A}] \hat{F}^{a b}(\boldsymbol{x}, \boldsymbol{y}) \rho^{b}(\boldsymbol{y}),
\end{aligned}
$$

where $\mathcal{J}[\boldsymbol{A}]$ is the determinant of the Faddeev-Popov operator, i.e. the inverse Coulomb ghost propagator

$$
\left(\hat{G}^{-1}\right)^{a b}(\boldsymbol{x}, \boldsymbol{y})=\left(-\partial_{i} \hat{D}_{i}^{a b}\right) \delta(\boldsymbol{x}-\boldsymbol{y}),
$$

${ }^{1}$ We will omit the index $\perp$ on transversal vector fields in the following. while the Coulomb Hamiltonian $H_{\mathrm{C}}$ describes the selfinteraction of non-Abelian color charges with density

$$
\rho^{a}(\boldsymbol{x})=\psi^{\dagger}(\boldsymbol{x}) T^{a} \psi(\boldsymbol{x})-f^{a b c} A_{i}^{b}(\boldsymbol{x}) \Pi_{i}^{c}(\boldsymbol{x})
$$

through the non-Abelian Coulomb kernel

$$
\hat{F}^{a b}(\boldsymbol{x}, \boldsymbol{y})=\int d^{3} z G^{a c}(\boldsymbol{x}, \boldsymbol{z})\left(-\partial_{\boldsymbol{z}}^{2}\right) G^{c b}(\boldsymbol{z}, \boldsymbol{y}) .
$$

The first term on the right-hand side of Eq. (5) is the matter charge density, which for the pure Yang-Mills case should be understood as an external source, while the second part is the dynamical charge density of the nonAbelian gauge field. In the Abelian theory the latter would, of course, be absent and Eq. (6) becomes the ordinary Coulomb kernel, i.e. the Green's function of the Laplacian $\hat{F}(\boldsymbol{x}, \boldsymbol{y})=(4 \pi|\boldsymbol{x}-\boldsymbol{y}|)^{-1}$.

From Eq. 6) one can define the non-Abelian color Coulomb potential, i.e. the Coulomb energy density for a pair of static quark-antiquark color charges separated by a distance $\boldsymbol{x}$ :

$$
V_{\mathrm{C}}^{a b}(\boldsymbol{p})=g^{2} \int d^{3} x e^{-i \boldsymbol{p} \cdot \boldsymbol{x}}\left\langle\hat{F}^{a b}(\boldsymbol{x}, \mathbf{0})\right\rangle .
$$

In a seminal paper 11 Zwanziger, extending ideas first put forward by Gribov 20, showed how such a Coulomb potential gives a natural upper bound to Wilson's physical potential [21. In other words, the presence of Coulomb confinement is a necessary condition for the physical confinement mechanism to take place in YangMills theories. These results are based on Gribov's intuition that the Yang-Mills dynamics must be restricted to the first Gribov region, where the Faddeev-Popov operator in Eq. (4) is positive definite ${ }^{2}$ Further signatures of this idea are the infra-red (IR) divergence of the Coulomb

\footnotetext{
${ }^{2}$ A unique elimination of all gauge copies requires an even further restriction to the so-called fundamental modular region, where the gauge functional only possesses absolute maxima.
} 
gauge ghost form factor and the emergence of an IR scale in the gluon dispersion relation [20, 22].

The Gribov-Zwanziger confinement scenario has been investigated in detail on the lattice 23 43, confirming the expected relationships between Coulomb gauge Greens-functions, Coulomb potential and confinement. However, since all lattice investigations are defined through an Euclidean-Lagrangian formalism, the contact with results obtained in a continuum Hamiltonian formulation is not straightforward. In particular, Weyl gauge can never be implemented on the lattice due to the periodic boundary conditions in the time direction, even in the so-called lattice Hamiltonian limit 41, 44, where one takes strongly anisotropic lattices with a much finer spacing in the temporal direction. At finite temperatures, due to the fixed finite length of the compactified time direction, the situation gets even worse.

The main problem is that in any Euclidean-Lagrangian formalism static quantities must be extracted from correlators which extend along the time direction ${ }^{3}$ Lattice Coulomb gauge observables, on the other hand, are defined at fixed time slices, involving only the space components of the vector fields. At $T=0$ the $O(4)$ rotational symmetry is unbroken and the restriction to space-like gauge fields is irrelevant, so that the Coulomb gauge analysis of confinement on the lattice is fully valid. As $T$ increases, however, it is conceivable that lattice Coulomb gauge observables, remaining "stuck" into the fixed time slice, will only sense space-space correlations. Since the area-law for spatial Wilson loops survives above $T_{c}$, causing screening effects in the quark gluon plasma, this non-perturbative effect could turn out to dominate the Coulomb gauge dynamics well above the deconfinement transition. In fact, all attempts to extend lattice investigations in Coulomb gauge to finite temperature [46] have up to now led to inconclusive results.

In this paper we give evidence that:

- on the lattice, the relationship between GribovZwanziger and Wilson confinement disappears above the deconfinement phase transition;

- the reason for such a failure lies in the strong correlation between the Coulomb string tension $\sigma_{C}$ and the spatial Wilson string tension.

Moreover, to calculate $\sigma_{C}$ one also needs to discretize the Coulomb potential. Contray to the continuum Hamiltonian formulation, on a finite lattice several inequivalent definitions of $V_{C}$ are possible. Our numerical results lead to the conclusion that extending the meaning of the Coulomb potential $V_{C}$ as the force between colour charges from the Hamiltonian picture to the lattice formulation can lead to inconsistent results and that the

\footnotetext{
${ }^{3}$ For instance, Polyakov loops are a most efficient way to determine the static inter-quark potential [45].
}

lattice versions of $V_{C}$ are sensitive to the same quark correlations that build the spatial string tension in the high temperature phase

To test our assumption, we need a tool to control the Wilson string tension $\sigma_{W}$, both for the whole ensemble and for its spatial directions separately. To do so, we adapt a method pioneered in Ref. [4] by either removing:

- all center vortices from the gauge field (full vortex removal);

- only vortices that pierce space-like Wilson loops (spatial vortex removal).

The rationale behind this strategy is clear: physical confinement should be caused by percolating center vortices piercing time-like Wilson loops [27, 36, 48, 51, 4 Removing all center vortices will thus disable confinement in the Yang-Mills ensemble, while removing spatial vortices only should therefore not affect the inter-quark potential; in fact, Polyakov loops correlators only involve temporal links and thus remain exactly unaffected by such a procedure. Any effect of spatial vortex removal on Coulomb gauge observables thus cannot be related to confinement and must, instead, be attributed to the disappearance of quark screening effects through the removal of the spatial string tension. This would then be a direct proof that such an observables predominantly see the spatial correlations in the gluon plasma rather than the confining force between static colour charges.

\section{SETUP}

\section{A. Lattice setup}

For our Coulomb gauge investigation we will employ the anisotropic Wilson action [44,66] for the colour group $S U(2)$ as proposed in Refs. [35, 40, 41]:

$$
\begin{aligned}
S & =\sum_{x}\left\{\beta_{s} \sum_{j>i=1}^{3}\left(1-\frac{1}{2} \operatorname{Retr}\left[U_{i j}(x)\right]\right)\right. \\
& \left.+\beta_{t} \sum_{i=1}^{3}\left(1-\frac{1}{2} \operatorname{Retr}\left[U_{i 4}(x)\right]\right)\right\},
\end{aligned}
$$

where $U_{\mu \nu}(x)$ is the standard plaquette. For each choice of $\beta_{s} \neq \beta_{t}$ the spatial and temporal lattice spacings $a_{s}$ and $a_{t}$ have to be determined non-perturbatively, giving the renormalized anisotropy through the ratio $\xi=a_{s} / a_{t}$. The couplings are usually parameterized as $\beta_{s}=\beta \gamma$ and $\beta_{t}=\beta / \gamma$, where $\gamma$ is the bare anisotropy, which needs

\footnotetext{
${ }^{4} \mathrm{~A}$ precise relationship between such a gauge-fixed, so called $\mathrm{P}$-vortices and the topological center vortices originally introduced by 't Hooft 52 is still missing. The interested reader is referred to Refs. [53 65] for further discussions on the subject.
} 
to be tuned with $\beta$ in order to realize the desired $\xi$ [44. Tables for $\xi$ and $a_{s}$ at selected choices of $\beta$ and $\gamma$ can be found, for the colour group $S U(2)$, in Ref. 41. All simulations for which no explicit value for $\xi$ is reported have been performed in the isotropic case $\xi=1$.

Most of the finite temperature simulations have been performed on lattices of sizes $V=N_{t} \times 32^{3}$ with varying $N_{t}$; different choices will be explicitly indicated in the data. The gluon propagator, the ghost propagator and the Coulomb potential have been computed from 100 independent samples in double precision, while the precise determination of the string tension through Creutz ratios at large distances required up to $10^{5}$ samples.

\section{B. Center vortex removal}

To identify center vortices, we first fixed the MCconfigurations to the direct maximal center gauge [50], i.e. we maximized

$$
F[U]=\sum_{x, \mu} \operatorname{tr}\left[U_{\mu}(x)^{2}\right]
$$

where $\mu=0,1,2,3$ for the full (standard) maximal center gauge and $\mu=1,2,3$ for the maximal center gauge restricted to the space-like links ("spatial maximal center gauge"). For the numerical implementation of Eq. (9) we have used an iterated over-relaxation algorithm based on the gauge-fixing CUDA code cuLGT 67]. For configurations which required subsequent Coulomb gauge fixing we stopped the center gauge fixing as soon as the functional value Eq. (9) changed by less than $\epsilon=10^{-12}$ within 100 iterations. For the measurements where no further gauge fixing was required we performed the center gauge fixing in single precision using $\epsilon=10^{-7}$.

Center projected configurations are then obtained after center gauge-fixing by mapping the links to the closest center element:

$$
Z_{\mu}^{\mathrm{s} / \mathrm{f}}(x)=\operatorname{sign} \operatorname{tr}\left[U_{\mu}(x)\right] \mathrm{I},
$$

where the index "s" and "f" stands for "spatial" and "full", respectively, with the index $\mu=1,2,3$ in the former and $\mu=0, \ldots, 3$ in the latter case. To create vortex free configurations, we follow Ref. [47] and define

$$
V_{\mu}^{\mathrm{s} / \mathrm{f}}(x)=Z_{\mu}^{\mathrm{s} / \mathrm{f}}(x) \cdot U_{\mu}(x),
$$

where $\mu$ runs again over only spatial or all Lorentz indices, respectively.

\section{Coulomb gauge}

Since we want to investigate the effect of vortex removal and center projection on correlators in Coulomb gauge, we need to transform each of the configurations
$\left\{Z^{\mathrm{f}}, Z^{\mathrm{s}}, V^{\mathrm{f}}, V^{\mathrm{s}}\right\}$ discussed above to Coulomb gauge ${ }^{5} \mathrm{We}$ employ a combination of simulated annealing and overrelaxation [75, 76, again adapting the CUDA code cuLGT 67]. For the center projected configurations we first had to apply a random gauge transformation, since the Coulomb FP-operator would otherwise be singular; the links in the center-projected, Coulomb gauge-fixed configurations are therefore no longer elements of $\mathbb{Z}_{2}$, but again of $S U(2)$.

After gauge-fixing we calculated, from the ghost propagator

$$
G(\boldsymbol{p})=\frac{d(\boldsymbol{p})}{|\boldsymbol{p}|^{2}}=\operatorname{tr}\left\langle(-\hat{\boldsymbol{D}} \cdot \nabla)^{-1}\right\rangle
$$

the ghost form factor $d(\boldsymbol{p})$ and the Coulomb potential

$$
V_{C}(\boldsymbol{p})=g^{2} \operatorname{tr}\left\langle(-\hat{\boldsymbol{D}} \cdot \nabla)^{-1}\left(-\nabla^{2}\right)(-\hat{\boldsymbol{D}} \cdot \nabla)^{-1}\right\rangle
$$

both directly in momentum space, where $(-\hat{\boldsymbol{D}} \cdot \nabla)$ is the Faddeev-Popov operator. If the Coulomb potential is linearly rising at large distances like $V_{C}(r) \simeq \sigma_{C} r$, its Fourier transform will behave as $V_{C}(p) \simeq 8 \pi \sigma_{C} / p^{4}$ at very small momenta. It is therefore customary to plot the quantity $p^{4} V_{C}(p)$ (often normalized by $8 \pi \sigma_{W}$ ) in which a non-zero intercept at $p \rightarrow 0$ signals a non-vanishing Coulomb string tension; we will follow this convention below.

As mentioned in the introduction, there is also an alternative definition of the Coulomb potential, directly calculated in position space:

$$
\begin{aligned}
a V_{C}(|\boldsymbol{x}-\boldsymbol{y}|) & =-\lim _{t \rightarrow 0} \frac{d}{d t} \log \left\langle\operatorname{tr}\left[P_{t}(\boldsymbol{x}) P_{t}^{\dagger}(\boldsymbol{y})\right]\right\rangle \\
& =-\log \left\langle\operatorname{tr}\left[U_{0}(\boldsymbol{x}) U_{0}^{\dagger}(\boldsymbol{y})\right]\right\rangle
\end{aligned}
$$

where $P_{t}(\boldsymbol{x})$ is a Polyakov line of length (at) starting at lattice site $(0, \boldsymbol{x})$. The equality in the second line is not obvious and discussed in more detail in Ref. [27, 38, 77]. Though formally equivalent in the Hamiltonian limit, such two alternative definitions of the Coulomb potential need not coincide on a finite lattice and will, in fact, show a rather different behaviour at finite temperatures. This is obviously due to the fact that Eq. (13) depends only on spatial links, whereas Eq. (14) depends only on temporal links; both get treated differently in Coulomb gauge and at finite temperature.

\footnotetext{
${ }^{5}$ We are aware that this procedure is not exactly equivalent to fixing Coulomb gauge directly. For a critical discussions about the effect of subsequent incomplete gauge fixings, Gribov copies and projections see e.g. Refs. 68 74.
} 


\section{RESULTS}

\section{A. Finite temperature in Coulomb gauge}

As discussed in the introduction, a coherent picture of the Gribov-Zwanziger confinement mechanism emerges from lattice Coulomb gauge investigations at $T=0$. As $T$ is increased, however, propagators do not seem to show a significant sensitivity to the deconfinement phase transition, as can be seen in Figs. 1, 2, 3, 4, for the gluon propagator, the ghost form factor and the Coulomb potential, respectively. Any deviation from the $T=0$ case starts well above $T=1.5 T_{c}$. Also, the non-trivial infrared behaviour seems to be at first enhanced, rather than decaying towards the perturbative expectation ${ }^{6}$ In particular, the Coulomb string tension extracted from the Coulomb potential in Figs. 3, 4 persists above the deconfinement phase transition, remaining constant up to $1.5 T_{c}$ and increasing above it; see the figure captions for further details.

These unexpected results were, in fact, the initial motivation for the present work. The fundamental puzzle is how it comes about that finite temperature correlators on the lattice decouple from the behaviour around $T_{c}$ expected from continuum investigations [16], while agreeing so well at $T=0$ ? Our working hypothesis is that the spatial rather than the temporal string tension underlies the finite temperature lattice Coulomb gauge dynamics. Indeed, the spatial string tension is known to persist and even rise above $T_{c}$, causing the strong correlations expected in the quark-gluon plasma. We have therefore decided to investigate this matter in more detail by going back to $T=0$ and controlling the string tension via the removal of (all or only spatial) center vortices in $\mathrm{MC}$ configurations.

\section{B. Vortex removal vs. Coulomb gauge}

\section{String tension}

In a first step, we calculated the temporal and spatial string tensions through Creutz ratios [78, defined at distance $R$ as in Ref. [79]

$$
\chi(T+0.5, R+0.5)=-\log \frac{W(T+1, R+1) W(T, R)}{W(T+1, R) W(T, R+1)} .
$$

To reduce the statistical noise we used 5 steps of APE smearing [80] with $\alpha=0.5$ for all links, or only for the spatial links (if only spatial vortices were removed); such

${ }^{6}$ The only exception might perhaps be he ghost form factor in Fig. 2. whose IR exponent seems to decrease for $T \gg T_{c}$; such an exponent is however quite difficult to determine reliably, see Ref. 41] for a critical discussion. a procedure cannot of course be applied to the center projected links.

We first calculated the spatial string tension at zero and finite temperatures to confirm that its dependence from the temperature mimics the one we found for the Coulomb potential in Figs. 3, 4. In Fig. 5 we show the spatial string tension for exactly the same configurations from which Figs. 3, 4 were calculated. The signal to noise ratio tends to get worse as $T$ and $r$ rise; the data for $T=$ $3 T_{c}$ above $r=7$ have been omitted, since their error-bars are orders of magnitude higher than the reported data. Still, it is obvious from the plot that, within errors, the spatial string tensions hardly changes up to $1.5 T_{c}$, while it is consistently higher at $3 T_{c}$. As a cross check, we have calculated the spatial string tension for a value of $\beta$ and at temperatures for which the signal to noise ratio is known to be good; results are shown in Fig. 6. The $T=$ 0 and the $T=1.1 T_{c}$ data are indistinguishable within error-bars; on the other hand, the $1.8 T_{c}$ data extrapolate to a higher spatial string tension, consistent with our expectations.

The above results show that we ought to be on a good path in assuming that the Coulomb and the spatial string tensions are correlated, since both behave semiquantitatively in the same way as the temperature is increased. We still however need to check the the spatial string tension is the "cause" for the Coulomb string tension. To achieve this, we will check what happens to the Coulomb string tension after removing degrees of freedom which are known to be strongly correlated to the confinement properties of the theory, namely center vortices.

As a first check we verified that, as expected from the literature, the Wilson string tension drops to zero after full vortex removal (VR) and, conversely, keeps its $S U(2)$ value after full center projection (CP) (see Fig. 7). Next, we repeat this procedure (vortex removal and center projection) in the spatial directions only. For the resulting configurations, it is necessary to distinguish between the temporal $\chi(T, R)$ and the spatial Creutz ratios $\chi\left(R_{1}, R_{2}\right)$. As expected, the spatial string tension $\sigma_{s}$ drops to zero after removing all spatial vortices, cf. Fig. 8. On the other hand, a direct measurement of the temporal string tension turns out to be impossible: as illustrated in the histogram in Fig. 9, all space-time Wilson loops receive random sign flips through spatial vortex removal; the signal to noise ratio becomes hopeless. However, the temporal string tension $\sigma_{t}$ measured from Polyakov loop correlators cannot change under spatial center projection $(\mathrm{sCP})$ or vortex removal $(\mathrm{sVR})$, since both procedures do not affect the temporal links from which the Polyakov lines are built. We can therefore safely conclude that all spatial vortex removed configurations are still confining, exhibiting the exact same value for $\sigma_{t}$ as the original, gauge-unfixed ones.

The spatial projection, with or without vortex removal, can further introduce gauge noise in the temporal links if followed by a Coulomb gauge fixing. This makes a di- 


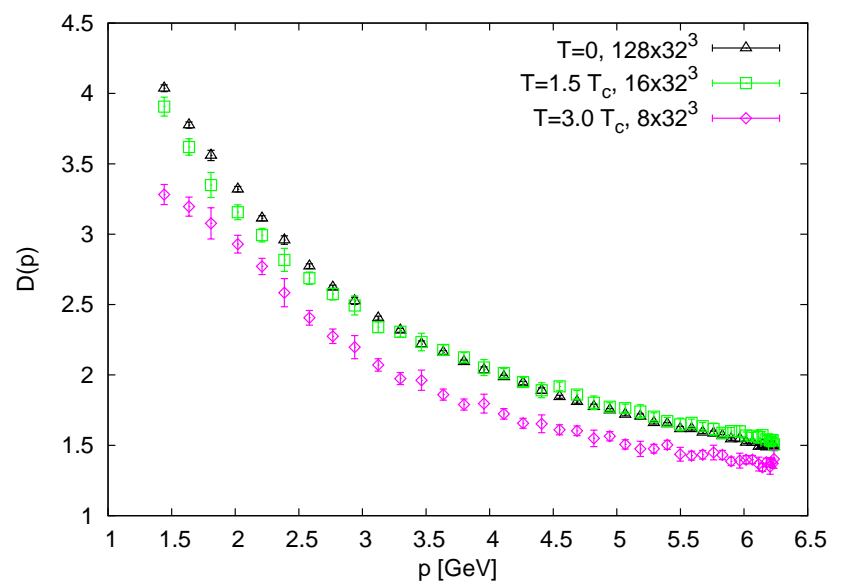

FIG. 1. Gluon propagator for various temperatures at $\beta=2.49$ and anisotropy $\xi=4$. As $T$ increases, the infrared Gribov mass $M \sim D^{-\frac{1}{2}}(0)$ increases as well [30, 33].

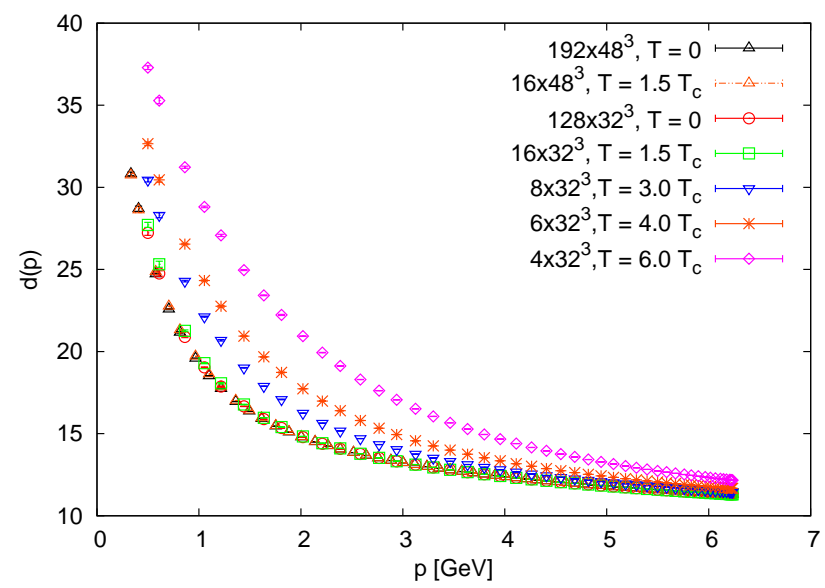

FIG. 2. Ghost form factor for various temperatures at $\beta=2.49$ and anisotropy $\xi=4$. No flattening is observed as $T$ is increased, except perhaps for the $T=6 T_{c}$ data (the upper curve), which displays a slight decrease in the exponent of the infrared increasing power law as compared to the other data.

rect measurement of the temporal string tension through Creutz-ratios challenging, as can be seen from the large error bars arising at large distances $r$ in Fig. 8 As can also be seen from this figure, both string tensions still exceed the asymptotic $S U(2)$ reference string tension at distances as large as $r \sim 9$, where they either have not yet reached a plateau (spatial) or are disappearing in statistical noise (temporal). We do not have a clear explanation for this slow convergence.

\section{Ghost form factor}

From the results above, it is obvious that the MC configurations after spatial vortex removal still exhibit temporal confinement but no spatial confinement. It is interesting to see how the Coulomb gauge correlators react to this change of physics in the underlying ensemble. As shown in Fig. 10, the ghost form factor is no longer compatible with a power law in the deep infrared, both after full and spatial vortex removal. As for the center projected configurations, a naive computation of ghost propagator is ill-defined because the Faddeev-Popov (FP) operator acquires additional zero modes from the center vortices which sit directly on the Gribov horizon: ${ }^{7}$ It is, however, possible to invert the FP operator in the subspace orthogonal to the kernel, which thus gives the subleading contributions to $d(\boldsymbol{p})$. The result is shown in Fig. 11, where we observe an enhancement in the midmomentum regime and a suppression in the deep infrared as compared to the unprojected Coulomb gauge. From these investigations, it is clear that the infra-red

\footnotetext{
${ }^{7}$ The $N_{c}^{2}-1$ constant zero modes are easy to take care of by restricting the calculation to momenta $p \neq 0$.
} 


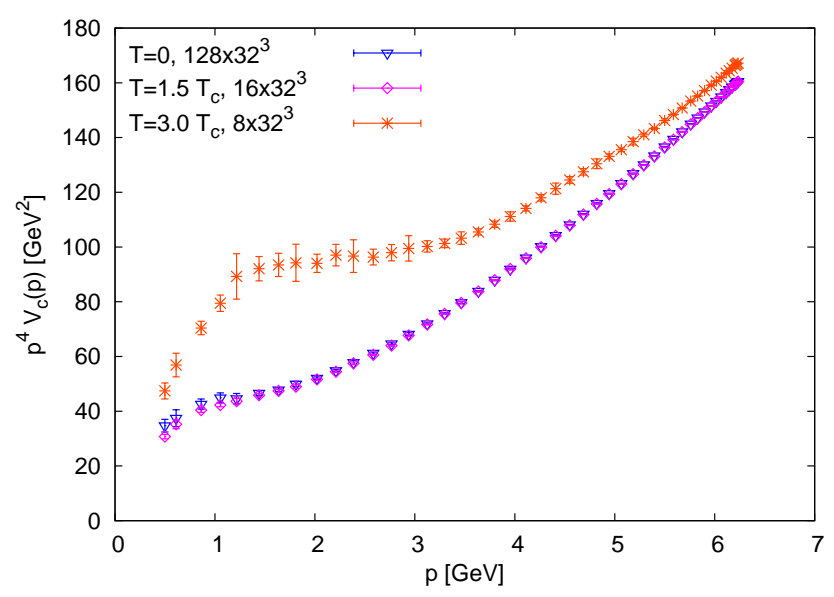

FIG. 3. Coulomb potential from Eq. 13 for various temperatures at $\beta=2.49$ and anisotropy $\xi=4$; the normalization is such that the intercept at $p \rightarrow 0$ equals $8 \pi \sigma_{C}$. We find that the extrapolation to $p \rightarrow 0$ is compatible with an increase of $\sigma_{C}$ at larger temperatures.

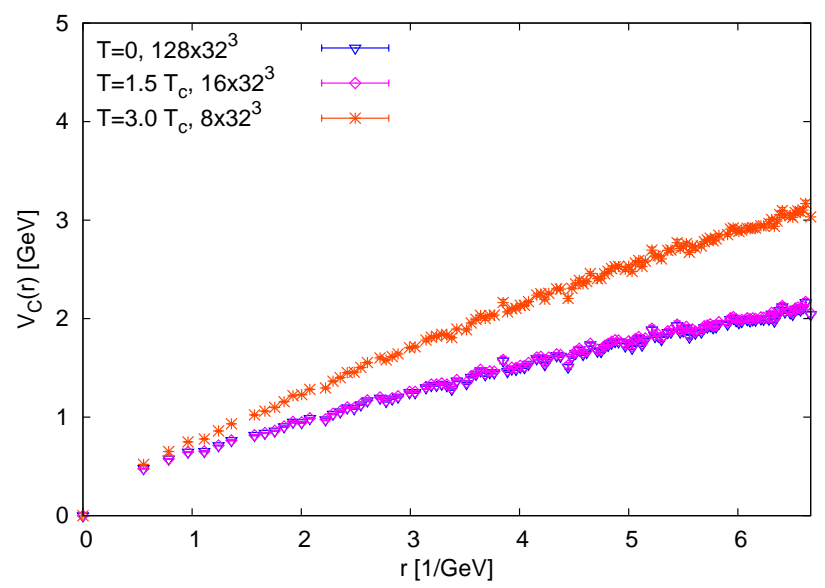

FIG. 4. Coulomb potential from Eq. (14) for various temperatures at $\beta=2.49$ and anisotropy $\xi=4$. The slope of the curve at large distances is obviously constant at least up to $1.5 T_{c}$ and increases at $3 T_{c}$.

enhancement of the original Coulomb gauge form factor is in fact tied to the spatial string tension, as elimination of the latter leads to to a dramatic suppression of the former in the IR.

\section{Coulomb potential}

The extrapolation of the Coulomb string tension $\sigma_{C}$ from the potential Eq. (13) is possible but suffers from large uncertainties for a variety of reasons. Estimates were given in Refs. [27, 29, 31, 34, 37, 41. We follow the convention in the literature and plot in Fig. 12 the ratio $p^{4} V_{C}(p) /\left(8 \pi \sigma_{W}\right)$, which yields $\sigma_{C} / \sigma_{W}$ in the limit $p \rightarrow 0$, cf. the remark after Eq. (13). As can be clearly seen from the plot, the Coulomb string tension $\sigma_{C}$ disappears after both full and spatial vortex removal. Since the latter case still contains the full temporal string tension, as discussed in Sec. IIIB 1, it is clear that the definition of the Coulomb string tension through Eq. 13 must be directly related to the spatial string tension.

It is interesting now to consider Eq. (14), our alternative definition of the Coulomb potential. From Ref. 27] this is known to allow for a better extrapolation of the Coulomb string tension while still vanishing after full vortex removal, cf. Fig. 13 , where it has been plotted together with its full center-projected and full vortex removed counterpart. On the other hand, the spatial vortex removed correlators must remain unchanged since Eq. (14) employs the temporal links $U_{0}(\boldsymbol{x})$ only. Remarkably, its spatial center projected counterpart still rises linearly, as it can be seen in Fig. 14. It is thus clear that the definition given in Eq. 14 of the Coulomb potential is indeed sensitive to both the temporal and the spatial string tension, as it still raises linearly both after spatial center projection and spatial vortex removal. This is not in contrast with the result found in Sec. III A above the phase transition the temporal string tension vanishes, 


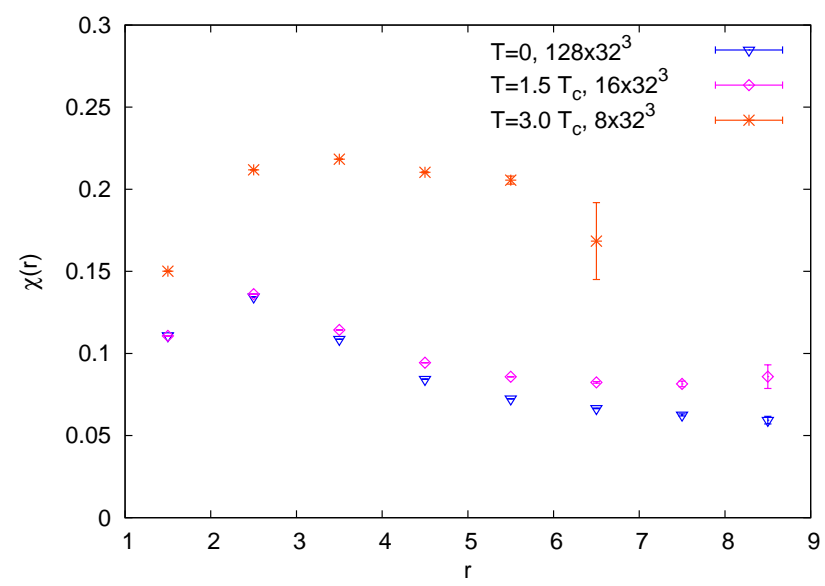

FIG. 5. Creutz ratios for the spatial string tension from the same configurations as in Figs. 3 , 4 , the dependence with the temperature is the same. Data at $3 T_{c}$ for $r>7$ have been omitted, since the signal to noise ratio was very poor.

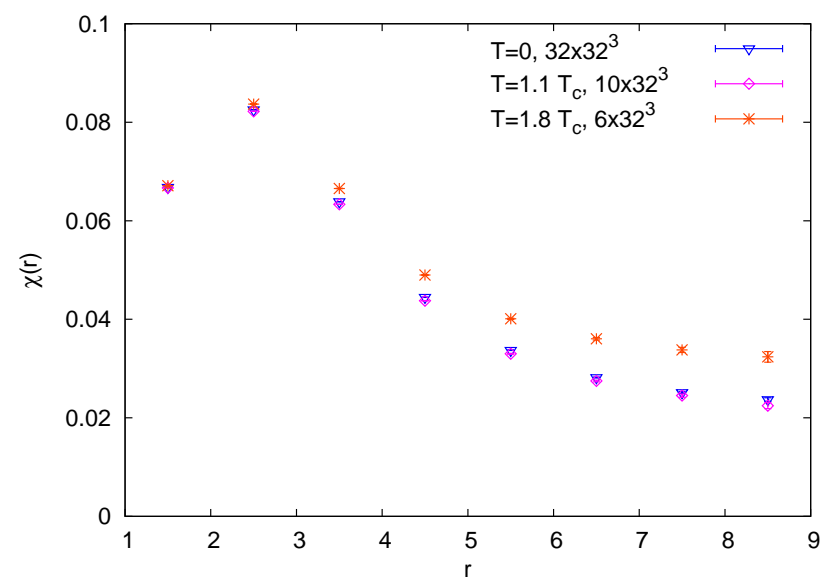

FIG. 6. Spatial string tension at $\beta=2.6$ for isotropic lattices up to a maximum temperature $1.8 T_{c}$.

thus leaving only the spatial string tension to affect the $U_{0}(\boldsymbol{x})$ correlators at high temperatures. Therefore, when either the temporal or the spatial string tension disappears, as above the deconfinement transiton (temporal), see Fig. 4, or after spatial vortex removal (spatial), see Fig. 14, Eq. (14) will still give a linear rising potential. Only when both are eliminated via full vortex removal, see Fig. 13 the $U_{0}(\boldsymbol{x})$ correlator becomes asimptotically flat.

Eq. (14) is therefore some kind of hybrid definition, sensitive to the confining string tension but, because of its "distance" to the Hamiltonian formulation due to being defined on a single time slice, still not sufficient to have overlap with all the excited states. The very large value it assumes after spatial center gauge fixing and projection (see Fig. 14) is likely a related phenomenon, since a mixing of degrees of freedom obviously occurs. Modifications of Eq. 14 might offer better results, since correlators of longer open Polyakov lines could turn out to be closer to the finite temperature dynamics in Coulomb gauge. However, as the length of the line increases, the relation- ship with the original Coulomb potential becomes obfuscated. Thus, a static Coulomb gauge observable that can detect the deconfinement phase transition on the lattice remains somewhat elusive.

\section{CONCLUSIONS}

In this paper we have investigated the relationship between spatial and Coulomb string tension as measured through the standard lattice definition of Coulomb gauge correlators. Such an observables are made out of the space-like links at a fixed time slice and, as we have seen, can only be used for investigations at $T=0$. As the temperature increases, temporal and spatial string tension decouple and we find that the dynamics of static Coulomb gauge observables are clearly dominated by the latter and not the former. This explains why the Coulomb string tension from Eq. (13) persists above $T_{c}$, and the low-order lattice Green's functions do not react 


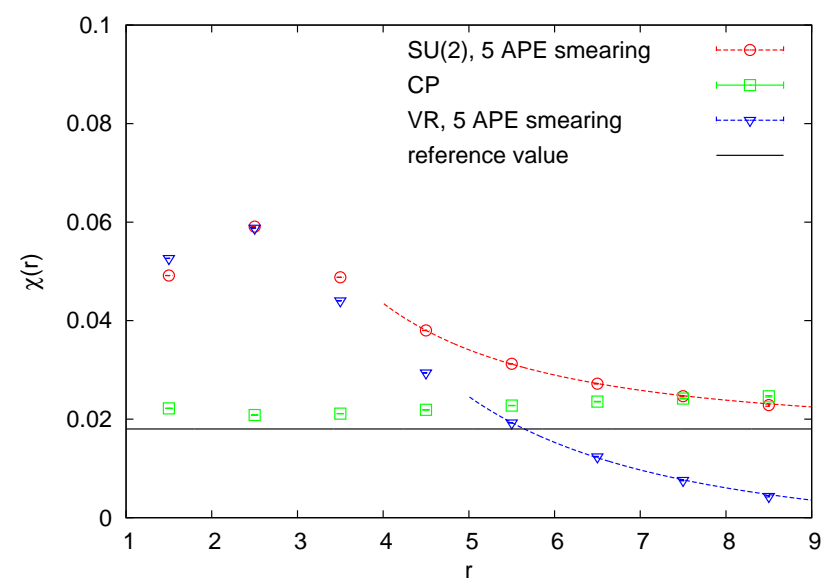

FIG. 7. Creutz ratios for full center vortex projection (CP) and removal (VR) at $\beta=2.6$. The reference value $\chi=0.01915$ was taken from Ref. [81]. Dotted lines are fits to the formula $\sigma+\frac{2 k}{r^{2}}$, see Ref. [79] for further details.

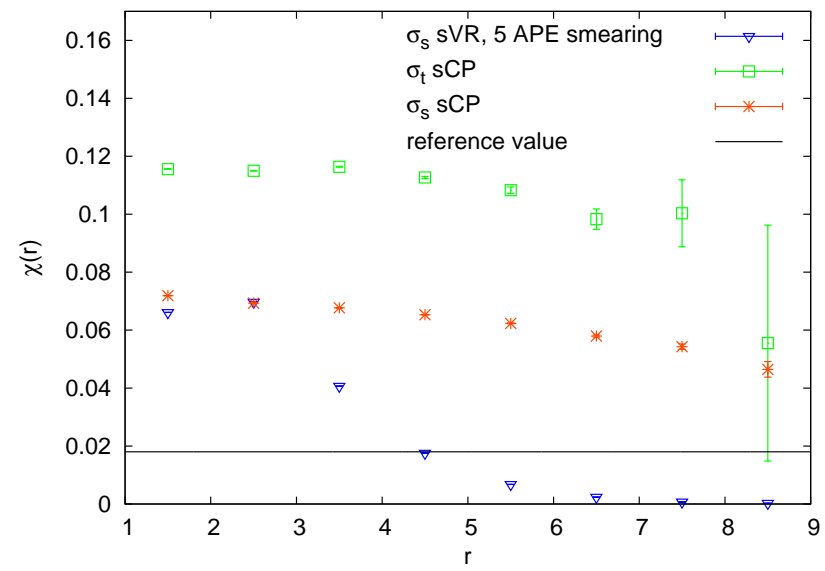

FIG. 8. Creutz ratios for spatial center vortex projection and removal at $\beta=2.6$, with the reference value taken from Ref. 81 . The symbols refer to the temporal $\left(\sigma_{t}\right)$ and spatial $\left(\sigma_{s}\right)$ string tension in the spatially center projected (sCP) and spatial vortex removed (sVR) ensemble, respectively.

to the loss of the temporal string tension at and above $T_{c}$.

The alternative definition of the Coulomb potential pioneered in Ref. [77, on the other hand, turns out to detect both the temporal and the spatial string tension, but it still cannot fully resolve the deconfinement phase transition, as this would probably require longer lines with temporal extensions comparable to the first excited states of the theory 27. Such an observables can, however, no longer be easily related to the static Coulomb potential.
More refined lattice observables are clearly necessary, and they may be tested with the methods layed out in this paper.

\section{ACKNOWLEDGMENTS}

This work was partially supported by the Deutsche Forschungsgemeinschaft under contract DFG-Re 856/91. H.V. wishes to thank the Evangelisches Studienwerk Villigst for financial support.
[1] D. Zwanziger, Phys.Rev.Lett. 90, 102001 (2003) arXiv:hep-lat/0209105 [hep-lat].

[2] R. Jackiw, I. Muzinich, and C. Rebbi, Phys. Rev. D17, 1576 (1978)

[3] D. Schutte, Phys.Rev. D31, 810 (1985).
[4] A. P. Szczepaniak and E. S. Swanson, Phys. Rev. D65, 025012 (2002), arXiv:hep-ph/0107078

[5] C. Feuchter and H. Reinhardt, Phys. Rev. D70, 105021 (2004), arXiv:hep-th/0408236

[6] W. Schleifenbaum, M. Leder, and H. Reinhardt, Phys. 


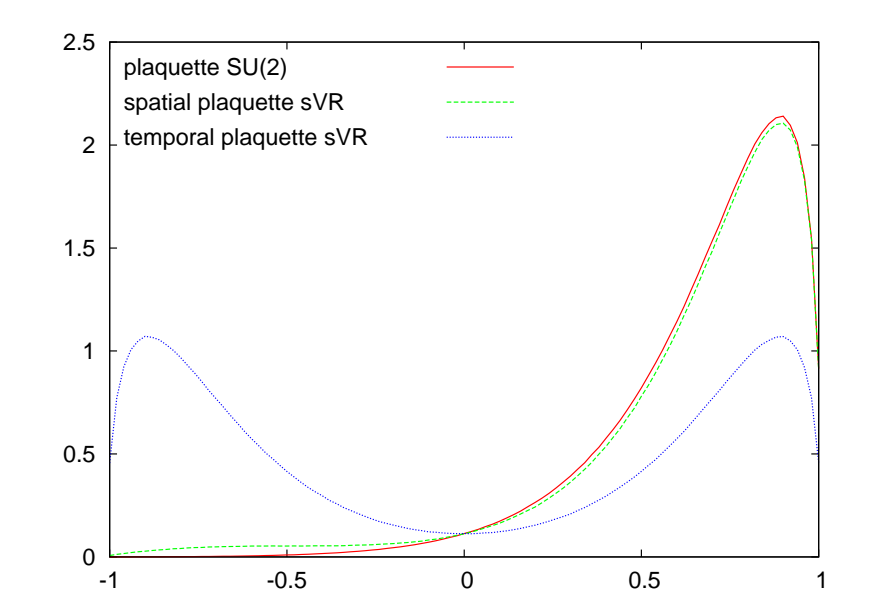

FIG. 9. Histogram of plaquettes before and after spatial vortex removal.

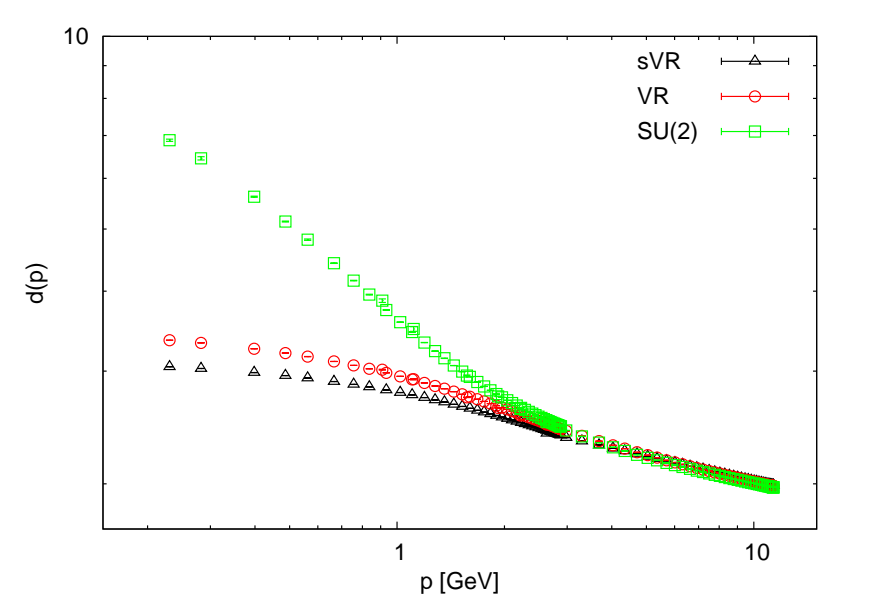

FIG. 10. Renormalized ghost form factor before and after vortex removal at $\beta=2.15$ and $\beta=2.60$. The data was multiplicatively renormalized at a reference scale of $\mu=3 \mathrm{GeV}$ whence the curves for the two couplings $\beta$ fall on top of each other.

Rev. D73, 125019 (2006), arXiv:hep-th/0605115

[7] D. Epple, H. Reinhardt, and W. Schleifenbaum, Phys. Rev. D75, 045011 (2007), arXiv:hep-th/0612241

[8] D. Epple, H. Reinhardt, W. Schleifenbaum, and A. P. Szczepaniak, Phys. Rev. D77, 085007 (2008), arXiv:0712.3694 [hep-th]

[9] C. Feuchter and H. Reinhardt, Phys. Rev. D77, 085023 (2008), arXiv:0711.2452 [hep-th].

[10] H. Reinhardt and W. Schleifenbaum, Annals Phys. 324, 735 (2009), arXiv:0809.1764 [hep-th]

[11] H. Reinhardt, Phys. Rev. Lett. 101, 061602 (2008), arXiv:0803.0504 [hep-th]

[12] D. R. Campagnari and H. Reinhardt, Phys.Rev. D82, 105021 (2010), arXiv:1009.4599 [hep-th]

[13] M. Pak and H. Reinhardt, Physics Letters B 707, 566 (2012), arXiv:1107.5263 [hep-ph]

[14] D. R. Campagnari and H. Reinhardt, Phys.Lett. B707, 216 (2012), arXiv:1111.5476 [hep-th]

[15] H. Reinhardt, D. Campagnari, and A. Szczepaniak, Phys.Rev. D84, 045006 (2011), arXiv:1107.3389 [hep-th]

[16] J. Heffner, H. Reinhardt, and D. R. Campagnari, Phys.Rev. D85, 125029 (2012), arXiv:1206.3936 [hep-th]
[17] H. Reinhardt and J. Heffner, Phys.Lett. B718, 672 (2012), arXiv:1210.1742 [hep-th]

[18] H. Reinhardt and J. Heffner, Phys.Rev. D88, 045024 (2013), arXiv:1304.2980 [hep-th]

[19] G. Burgio, R. De Pietri, H. A. Morales-Tecotl, L. F. Urrutia, and J. D. Vergara, Nucl. Phys. B566, 547 (2000), arXiv:hep-lat/9906036.

[20] V. N. Gribov, Nucl. Phys. B139, 1 (1978)

[21] K. G. Wilson, Phys.Rev. D10, 2445 (1974)

[22] J. M. Cornwall, Nucl. Phys. B157, 392 (1979).

[23] D. Zwanziger, Nucl.Phys. B412, 657 (1994)

[24] D. Zwanziger, Nucl. Phys. B485, 185 (1997), arXiv:hepth/9603203

[25] A. Cucchieri and D. Zwanziger, Phys. Rev. D65, 014001 (2002), arXiv:hep-lat/0008026.

[26] A. Cucchieri and D. Zwanziger, Phys. Lett. B524, 123 (2002), arXiv:hep-lat/0012024.

[27] J. Greensite and S. Olejnik, Phys.Rev. D67, 094503 (2003), arXiv:hep-lat/0302018 [hep-lat]

[28] K. Langfeld and L. Moyaerts, Phys. Rev. D70, 074507 (2004), arXiv:hep-lat/0406024

[29] J. Greensite, S. Olejnik, and D. Zwanziger, Phys.Rev. 


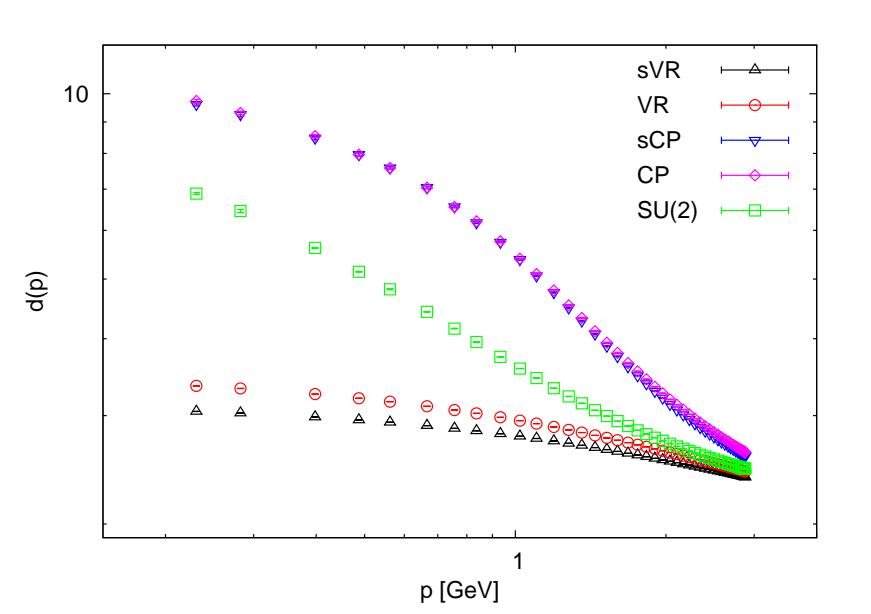

FIG. 11. Ghost form factor after center projection (and restriction to the non-zero subspace) at $\beta=2.15$.

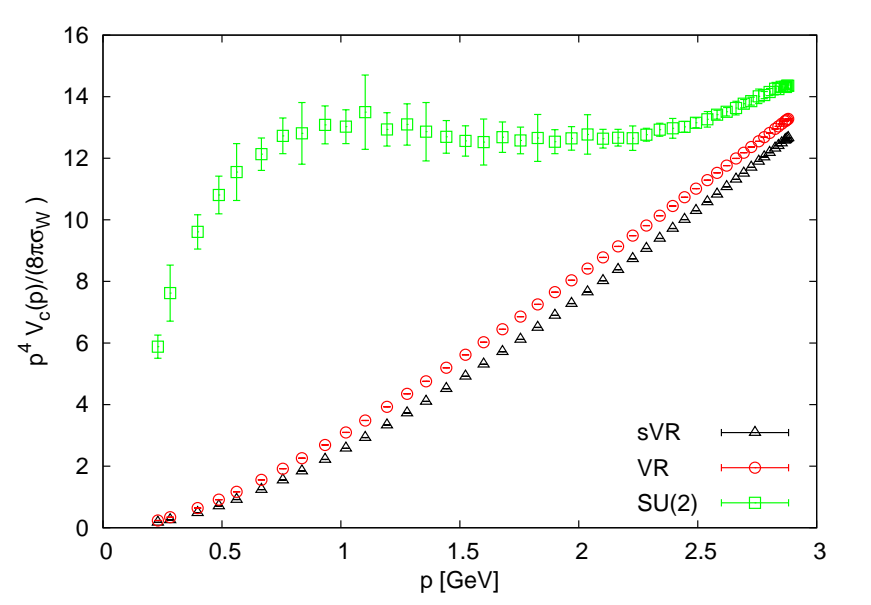

FIG. 12. Standard Coulomb potential Eq. 13 in momentum space, normalized such that the intercept at $p \rightarrow 0$ yields $8 \pi \sigma_{C}$ The relatively sharp drop in the deep infrared for the full $S U(2)$ data made it necessary to take a fairly small coupling $\beta=2.15$ to (roughly) estimate the intercept.

D69, 074506 (2004), arXiv:hep-lat/0401003 [hep-lat].

[30] G. Burgio, M. Quandt, and H. Reinhardt, Phys. Rev. Lett. 102, 032002 (2009), arXiv:0807.3291 [hep-lat].

[31] A. Voigt, E.-M. Ilgenfritz, M. Muller-Preussker, and A. Sternbeck, Phys.Rev. D78, 014501 (2008) arXiv:0803.2307 [hep-lat]

[32] J. Greensite, Phys.Rev. D80, 045003 (2009), arXiv:0903.2501 [hep-lat]

[33] G. Burgio, M. Quandt, and H. Reinhardt, Phys.Rev. D81, 074502 (2010), arXiv:0911.5101 [hep-lat]

[34] Y. Nakagawa, A. Nakamura, T. Saito, and T. Toki, Mod.Phys.Lett. A23, 2348 (2008)

[35] Y. Nakagawa, A. Nakamura, T. Saito, and H. Toki, PoS LAT2009, 230 (2009), arXiv:0911.2550 [hep-lat]

[36] M. Quandt, H. Reinhardt, and G. Burgio, Phys.Rev. D81, 065016 (2010), arXiv:1001.3699 [hep-lat].

[37] Y. Nakagawa, A. Nakamura, T. Saito, and H. Toki, Phys.Rev. D81, 054509 (2010), arXiv:1003.4792 [heplat]

[38] T. Iritani and H. Suganuma, Phys.Rev. D83, 054502 (2011), arXiv:1011.4767 [hep-lat]
[39] H. Reinhardt, M. Quandt, and G. Burgio, Phys. Rev. D 85, 025001 (2012), arXiv:1110.2927 [hep-th]

[40] Y. Nakagawa, A. Nakamura, T. Saito, and H. Toki, Phys.Rev. D83, 114503 (2011), arXiv:1105.6185 [heplat]

[41] G. Burgio, M. Quandt, and H. Reinhardt, Phys.Rev. D86, 045029 (2012), arXiv:1205.5674 [hep-lat]

[42] G. Burgio, M. Schrock, H. Reinhardt, and M. Quandt, Phys.Rev. D86, 014506 (2012), arXiv:1204.0716 [heplat]

[43] T. Iritani and H. Suganuma, Phys.Rev. D86, 074034 (2012), arXiv:1204.6591 [hep-lat]

[44] G. Burgio and al. (TrinLat), Phys. Rev. D67, 114502 (2003), arXiv:hep-lat/0303005

[45] M. Luscher and P. Weisz, JHEP 0109, 010 (2001), arXiv:hep-lat/0108014 [hep-lat].

[46] H. Vogt, G. Burgio, M. Quandt, and H. Reinhardt, PoS LATTICE2013, 363 (2014), arXiv:1311.5707 [hep-lat]]

[47] P. de Forcrand and M. D'Elia, Phys.Rev.Lett. 82, 4582 (1999), arXiv:hep-lat/9901020 [hep-lat]

[48] L. Del Debbio, M. Faber, J. Greensite, and S. Ole- 


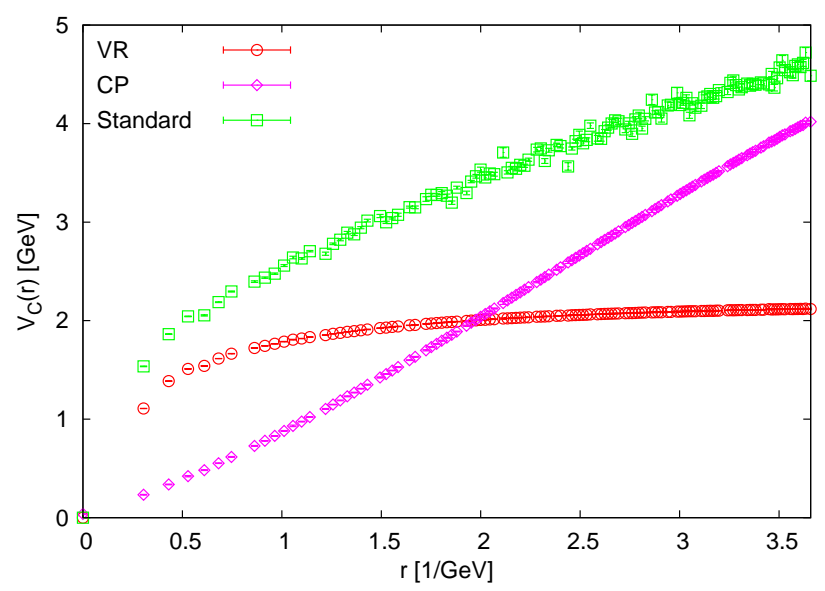

FIG. 13. Coulomb potential from Eq. (14) in position space. Linear behaviour sets in at moderate distances $r$, so that a fairly large coupling $\beta=2.60$ could be afforded to minimize discretization effects. See the discussion at the end of section IIIB for further details.

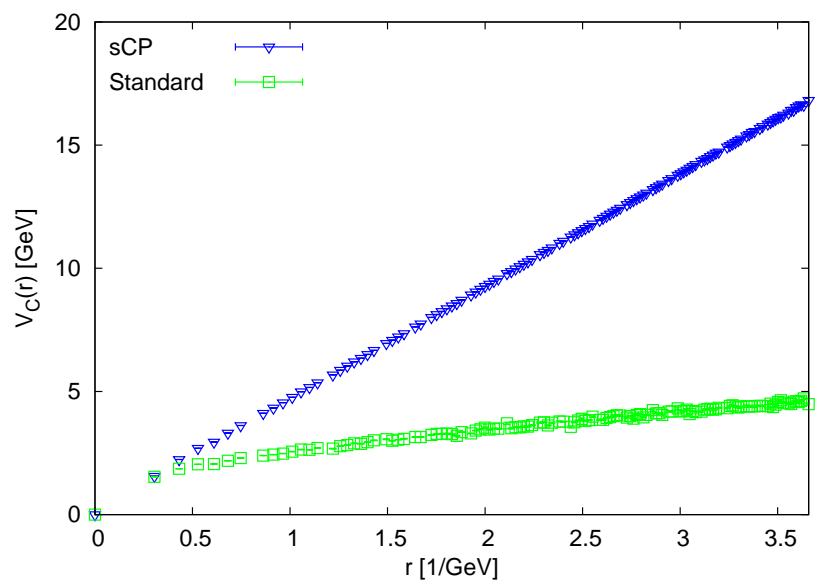

FIG. 14. Coulomb potential from Eq. (14) in position space. Both the spatial center projected and the spatial vortex removed correlators are compatible with a linear rising potential, although the former overestimates the string tension by a large factor.

jnik, Phys.Rev. D55, 2298 (1997) arXiv:hep-lat/9610005 [hep-lat]

[49] K. Langfeld, H. Reinhardt, and O. Tennert, Phys.Lett. B419, 317 (1998), arXiv:hep-lat/9710068 [hep-lat]

[50] L. Del Debbio, M. Faber, J. Giedt, J. Greensite, and S. Olejnik, Phys.Rev. D58, 094501 (1998), arXiv:heplat/9801027 [hep-lat].

[51] M. Engelhardt, K. Langfeld, H. Reinhardt, and O. Tennert, Phys.Rev. D61, 054504 (2000), arXiv:heplat/9904004 [hep-lat]

[52] G. 't Hooft, Nucl.Phys. B153, 141 (1979)

[53] A. Hart, B. Lucini, Z. Schram, and M. Teper, JHEP 0006, 040 (2000), arXiv:hep-lat/0005010 [hep-lat]

[54] T. G. Kovacs and E. Tomboulis, Phys.Rev.Lett. 85, 704 (2000), arXiv:hep-lat/0002004 [hep-lat]

[55] P. de Forcrand, M. D'Elia, and M. Pepe, Phys.Rev.Lett. 86, 1438 (2001), arXiv:hep-lat/0007034 [hep-lat].

[56] A. Barresi, G. Burgio, and M. Muller-Preussker, Nucl.Phys.Proc.Suppl. 106, 495 (2002), arXiv:heplat/0110139 [hep-lat]

[57] P. de Forcrand and L. von Smekal, Phys.Rev. D66,
011504 (2002) arXiv:hep-lat/0107018 [hep-lat]

[58] A. Barresi, G. Burgio, and M. Muller-Preussker, Nucl.Phys.Proc.Suppl. 119, 571 (2003), arXiv:heplat/0209011 [hep-lat].

[59] H. Reinhardt, Phys.Lett. B557, 317 (2003), arXiv:hepth/0212264 [hep-th]

[60] A. Barresi, G. Burgio, and M. Muller-Preussker, Phys.Rev. D69, 094503 (2004), arXiv:hep-lat/0309010 [hep-lat]

[61] A. Barresi, G. Burgio, M. D'Elia, and M. MullerPreussker, Phys.Lett. B599, 278 (2004), arXiv:heplat/0405004 [hep-lat]

[62] A. Barresi and G. Burgio, Eur.Phys.J. C49, 973 (2007) arXiv:hep-lat/0608008 [hep-lat]

[63] G. Burgio, M. Fuhrmann, W. Kerler, and M. MullerPreussker, Phys.Rev. D74, 071502 (2006), arXiv:hepth/0608075 [hep-th]

[64] G. Burgio, M. Fuhrmann, W. Kerler, and M. MullerPreussker, Phys.Rev. D75, 014504 (2007), arXiv:heplat/0610097 [hep-lat].

[65] G. Burgio and H. Reinhardt, Phys.Rev. D91, 025021 
(2015), arXiv:1412.1762 [hep-lat]

[66] T. R. Klassen, Nucl.Phys. B533, 557 (1998), arXiv:heplat/9803010 [hep-lat]

[67] M. Schröck and H. Vogt, Comput.Phys.Commun. 184, 1907 (2013), arXiv:1212.5221 [hep-lat]

[68] G. Bali, V. Bornyakov, M. Muller-Preussker, and K. Schilling, Phys.Rev. D54, 2863 (1996), arXiv:heplat/9603012 [hep-lat]

[69] V. Bornyakov, D. Komarov, and M. Polikarpov, Phys.Lett. B497, 151 (2001), arXiv:hep-lat/0009035 [hep-lat]

[70] L. Giusti, M. Paciello, C. Parrinello, S. Petrarca, and B. Taglienti, Int.J.Mod.Phys. A16, 3487 (2001) arXiv:hep-lat/0104012 [hep-lat],

[71] M. Faber, J. Greensite, and S. Olejnik, Phys.Rev. D64, 034511 (2001), arXiv:hep-lat/0103030 [hep-lat]

[72] M. Faber, J. Greensite, and S. Olejnik, JHEP 0111, 053 (2001), arXiv:hep-lat/0106017 [hep-lat]

[73] P. Y. Boyko, V. Bornyakov, E.-M. Ilgenfritz, A. Kovalenko, B. Martemyanov, et al., Nucl.Phys. B756, 71 (2006), arXiv:hep-lat/0607003 [hep-lat]
[74] A. O'Cais, W. Kamleh, K. Langfeld, B. Lasscock, D. Leinweber, et al., Phys.Rev. D82, 114512 (2010) arXiv:0807.0264 [hep-lat].

[75] I. Bogolubsky, G. Burgio, M. Muller-Preussker, and V. Mitrjushkin, Phys.Rev. D74, 034503 (2006) arXiv:hep-lat/0511056 [hep-lat]

[76] I. Bogolubsky, V. Bornyakov, G. Burgio, E. Ilgenfritz, M. Muller-Preussker, et al., Phys.Rev. D77, 014504 (2008), arXiv:0707.3611 [hep-lat].

[77] E. Marinari, M. L. Paciello, G. Parisi, and B. Taglienti, Phys.Lett. B298, 400 (1993), arXiv:hep-lat/9210021 [hep-lat]

[78] M. Creutz, Phys.Rev.Lett. 45, 313 (1980)

[79] A. Gonzalez-Arroyo and M. Okawa, Phys.Lett. B718, 1524 (2013), arXiv:1206.0049 [hep-th]

[80] M. Albanese et al. (APE Collaboration), Phys.Lett. B192, 163 (1987)

[81] J. C. R. Bloch, A. Cucchieri, K. Langfeld, and T. Mendes, Nucl. Phys. B687, 76 (2004), arXiv:heplat/0312036 